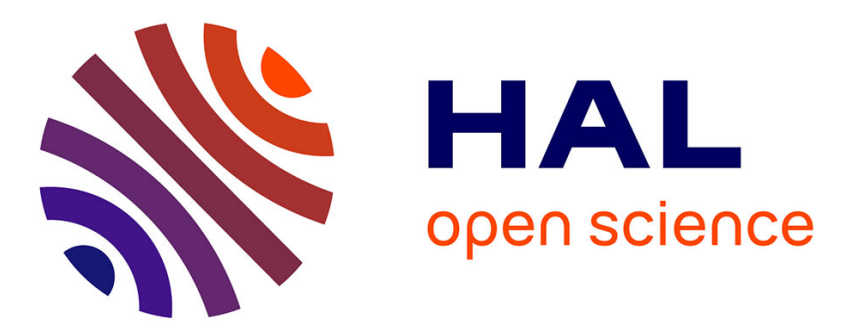

\title{
Boundary conditions at a fluid-porous interface: An a priori estimation of the stress jump coefficients
}

\author{
M. Chandesris, D. Jamet
}

\section{To cite this version:}

M. Chandesris, D. Jamet. Boundary conditions at a fluid-porous interface: An a priori estimation of the stress jump coefficients. International Journal of Heat and Mass Transfer, 2006, 49 (13-14), pp.2137-2150. 10.1016/j.ijheatmasstransfer.2005.12.010 . cea-02354114

\section{HAL Id: cea-02354114 https://hal-cea.archives-ouvertes.fr/cea-02354114}

Submitted on 14 Nov 2019

HAL is a multi-disciplinary open access archive for the deposit and dissemination of scientific research documents, whether they are published or not. The documents may come from teaching and research institutions in France or abroad, or from public or private research centers.
L'archive ouverte pluridisciplinaire HAL, est destinée au dépôt et à la diffusion de documents scientifiques de niveau recherche, publiés ou non, émanant des établissements d'enseignement et de recherche français ou étrangers, des laboratoires publics ou privés. 


\title{
Boundary conditions at a planar fluid-porous interface for a Poiseuille flow
}

\author{
M. Chandesris ${ }^{a}$, D. Jamet ${ }^{a}$ * \\ ${ }^{a}$ Laboratoire de Modélisation et Développement Logiciels, DEN/DER/SSTH, CEA Grenoble, \\ 17 rue des Martyrs, 38054 Grenoble Cedex 9, France.
}

\begin{abstract}
The velocity boundary condition that must be imposed at an interface between a porous medium and a free fluid is investigated. A heterogeneous transition zone characterized by rapidly varying properties is introduced between the two homogeneous porous and free fluid regions. The problem is solved using the method of matched asymptotic expansions and boundary conditions between the two homogeneous regions are obtained. The continuity of the velocity is recovered and a jump in the stress built using the viscosity (and not the effective viscosity) appears. This result also provides an explicit dependence of the stress jump coefficient to the internal structure of the transition zone and its sensitivity to this micro structure is recovered.
\end{abstract}

Keywords: Interface, Boundary conditions, Porous media, Incompressible fbw, Matched asymptotic expansions.

\section{Introduction}

Transport phenomena at the interface between a porous medium and an adjacent free fluid have been the object of considerable interest because they occur in a wide range of technological applications. In all problems, the knowledge of the momentum transfer across the interface is decisive for an accurate description of the other involved transfer phenomena: heat, species,... The main modeling issues remain in the definition of appropriate boundary conditions at the fluid/porous interface, as well as in the choice of the appropriate model in the porous region.

In the study of Beavers and Joseph [1], which is recalled in figure 1, the momentum transport in the free flid region is described by the Stokes equations, while the Darcy's law

$$
-\frac{\mu}{K_{p}}\langle u\rangle=\frac{d\langle p\rangle_{f}}{d x}
$$

is used in the porous medium. Here, $\langle u\rangle$ is the volumetric average of the velocity, $\mu$ is the flid dynamic viscosity, $K_{p}$ is the permeability of the porous medium and $\langle p\rangle_{f}$ denotes the intrinsic average of the pressure. Since the Stokes and Darcy equations are partial differential equations of a different order, a simple semi-empirical slip boundary condition is proposed at the interface, which takes into account the Darcy velocity inside the porous layer $\left(U_{D}\right)$, the permeability of the porous medium $\left(K_{p}\right)$ and a slip parameter $\alpha$ :

$$
\left.\frac{d u}{d y}\right|_{y=0}=\frac{\alpha}{\sqrt{K_{p}}}\left(u_{B}-U_{D}\right)
$$

where $u_{B}=\left.\langle u\rangle\right|_{y=0}$ is the free fluid velocity at the interface (slip velocity). $\alpha$ is a dimensionless constant which characterizes the geometry of the transition region between the free fluid region and the porous medium. This constant needs to be adjusted to obtain good agreement with the experimental data provided by Beavers and Joseph [1].

Neale and Nader [11] proposed to use the Darcy-Brinkman equations

$$
\mu_{\mathrm{eff}} \frac{d^{2}\langle u\rangle}{d y^{2}}-\frac{\mu}{K_{p}}\langle u\rangle=\frac{d\langle p\rangle_{f}}{d x}
$$

for the fbw inside the porous medium in order to explain the semi-empirical theory of Beavers and Joseph. Here, $\mu$ eff denotes the effective viscosity of the fluid in the porous medium. Since the Stokes and Brinkman equations are of

\footnotetext{
${ }^{*}$ Corresponding author. Tel.: +33-4-38-78-45-42; fax: +33-4-38-78-51-95. E-mail address: didier.jamet@cea.fr
} 


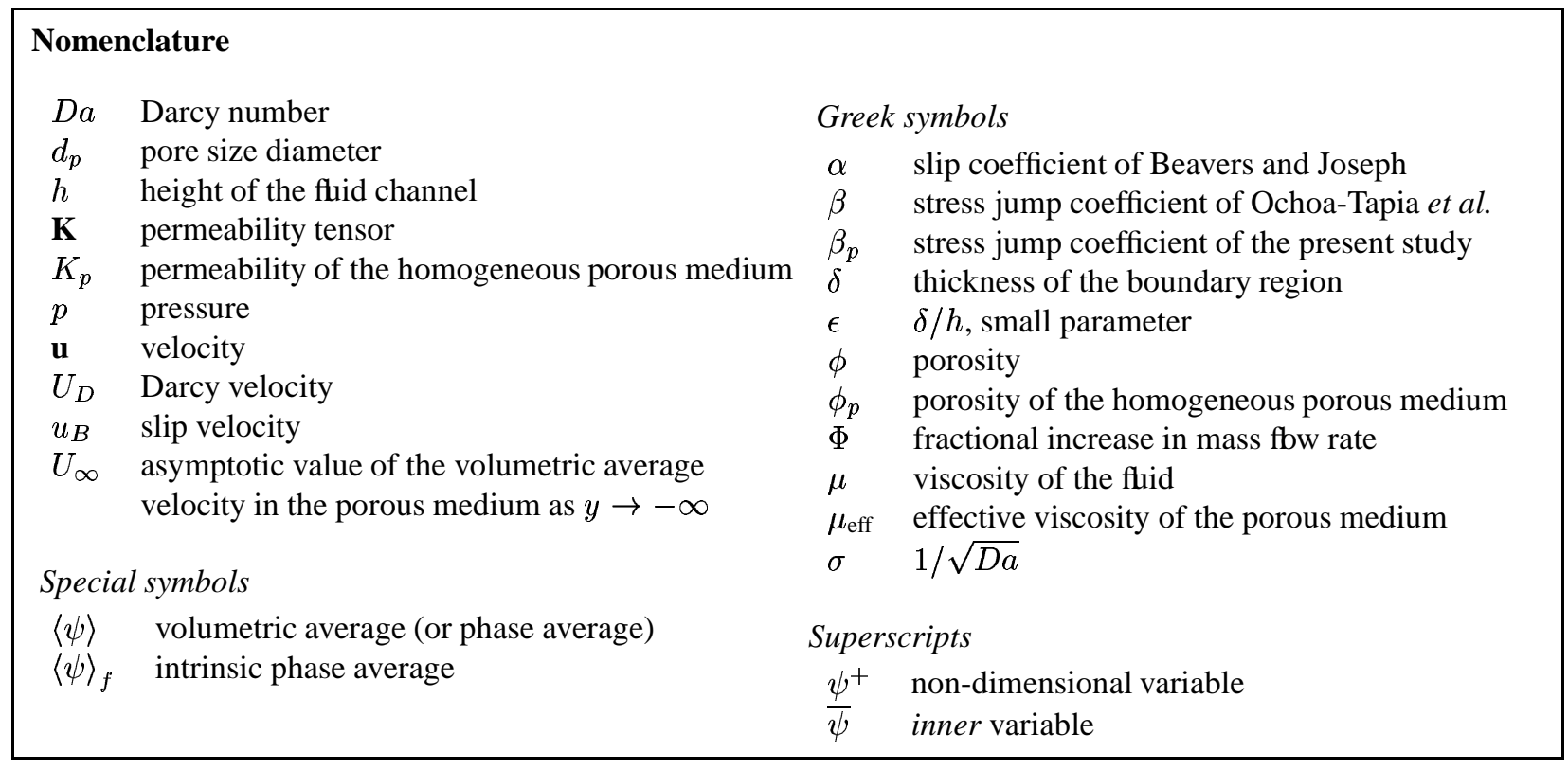

the same order, they assumed continuity of both velocity and stress (built on the effective viscosity) at the interface. In that case, they obtained a solution identical to the one of Beavers and Joseph in the free fluid region $(y>0)$ provided that $\alpha=\sqrt{\mu_{\text {eff }} / \mu}$. With this formulation, it is possible to obtain information about what is happening in the boundary layer region beneath the surface of the permeable channel wall and some authors use this formulation $[19,16,25]$. The main limitation of this approach lies in the fact that it is still not possible to predict accurately the effective viscosity $\mu_{\text {eff }}$ of a given porous medium. In fact, even the validity of the Brinkman correction, in which the effective viscosity appears, is challenged [23, 12,24]. Furthermore, Sahraoui and Kaviany [21] have shown that with this kind of formulation (i.e. Brinkman and Stokes), a variable effective viscosity has to be used for an accurate prediction of the local velocity near the interface inside the porous medium.

An alternative approach has been proposed by Ochoa-Tapia and Whitaker [13, 14], who developed a jump condition to account for the momentum transfer that occurs at the interface. Their work is based on the continuity of volume averaged quantities in the entire domain. They use volume averaged transport equations in the whole domain, including the heterogeneous transition region and propose a methodology to derive the corresponding jump conditions to be applied at the interface between the two homogeneous region. They obtain the following jump condition:

$$
\left.\frac{d\langle u\rangle}{d y}\right|_{y=0^{+}}-\left.\frac{1}{\phi_{p}} \frac{d\langle u\rangle}{d y}\right|_{y=0^{-}}=-\left.\frac{\beta}{\sqrt{K_{p}}}\langle u\rangle\right|_{y=0}
$$

where $\beta$ is a dimensionless parameter of the order of one. They obtain good agreement with the experimental data of Beavers and Joseph [1] by adjusting this parameter. In their analysis, $\beta$ is a complex function of a closure problem that they could not solve, even numerically. Thus, they propose to use a variable porosity model in a transition zone between the two homogeneous regions, as a substitute for the jump condition to try to make explicit the dependence of $\beta$ with the structure of this transition zone. However, their approach was not successful.

Following the same idea, Goyeau et al. [7] recall that "an interface is an ideal representation of a region with continuous spatial changes of the macroscopic properties and that the knowledge of these evolving heterogeneities is necessary for an accurate description of transport phenomena near the interface". Thus, they introduce a heterogeneous continuously varying transition zone between the two outer homogeneous regions. They are then able to obtain an explicit function for the stress jump coefficient $\beta$ and they show that $\beta$ is related to the continuous spatial variations of the porous structure within the transition zone. The main limitation of their approach lies in the fact that $\beta$ is also related to the variations of the velocity, which is an unknown of the problem.

In order to study this problem, different levels of description can be considered. At the microscopic level, the $\mathrm{fbw}$ is governed by the Stoke equations in the free flid channel and inside the pores of the permeable media. At a second level of description, the fluid and solid phases are replaced by an equivalent medium obtained by using the volume averaging method. This is the basis of the continuum approach for fbws in porous media. At this continuous level, the zone located in between the two homogeneous regions (i.e. porous medium and free fluid), is a continuous heterogeneous transition zone, where the properties of the medium (e.g. porosity) encounter strong but nevertheless continuous variations. Finally, a third level of description characterized by two homogeneous regions, separated by an interface of discontinuity can be considered. The use of a single volume averaged transport equation in the whole 
domain, as well as the representation of this problem using a heterogeneous continuously varying transition zone of thickness $\delta$ between two outer homogeneous regions (second level approach) are attractive. Goyeau et al. [7] could not give an expression for $\beta$ that does not depend on the variations of the velocity because they do not explicitly solve the problem inside the heterogeneous transition zone. The objective of our study is to show that, given a transport equation in the whole domain (second level of description), it is possible to solve the problem inside the heterogeneous transition zone using the method of matched asymptotic expansions and thus to formally derive the boundary conditions to be applied at the interface separating the two homogeneous regions (third level of description). An explicit function for the stress jump coefficient which only depends on the characteristics of the porous medium (porosity and permeability) in the transition zone is thus obtained.

In the first part of this paper, the derivation of the macroscopic continuity and momentum equations in the framework of the volume averaging method are recalled, and the hypothesis used in this study are presented. Then, the problem is solved using the method of matched asymptotic expansions and boundary conditions at the interface are derived. Finally, the result is compared to the experimental data of Beavers and Joseph [1], and to the analytical solution of Ochoa-Tapia and Whitaker [14].

\section{Macroscopic governing equations}

\subsection{Volume averaging}

The method of volume averaging is a technique used to rigorously derive continuous macroscopic equations from the description of the problem at a microscopic scale for multiphase systems. This method allows to change the scale of description of the problem. The main steps of the method will be described, for completeness and to highlight the hypotheses used in this study.

For the problem under consideration in the present study, the fbw is laminar. The Reynolds number inside the pores is supposed to be small, so that inertia effects can be neglected. The governing differential equations at the microscopic scale in the entire fluid domain (free fluid region and pores of the porous medium) are given by the Stokes equations:

$$
\begin{aligned}
& \nabla \cdot \mathbf{u}=0 \\
& -\nabla p+\mu \nabla^{2} \mathbf{u}=0
\end{aligned}
$$

The boundary condition is:

$$
\mathbf{u}=0 \quad \text { at the fluid-solid interface }
$$

The macroscopic governing equations can be obtained by volume averaging the corresponding microscopic equations (5)-(6) over a representative elementary volume (REV). In the study of multiphase transport phenomena, two types of volume averages are commonly introduced [27]. The first one is the phase average (or volumetric average) which is defined by:

$$
\langle\psi\rangle=\frac{1}{V} \int_{V_{f}} \psi d V
$$

for any physical variable $\psi$, where $V_{f}$ represents the volume of the fluid phase contained within the averaging volume $V$ (see figure 1). However, $\langle\psi\rangle$ is not equal to $\psi$ when the latter is a constant. Therefore a second average, the intrinsic phase average, is defined by:

$$
\langle\psi\rangle_{f}=\frac{1}{V_{f}} \int_{V_{f}} \psi d V
$$

which is more representative of the condition in the fluid phase. These two averages are related through the porosity $\phi$ by:

$$
\langle\psi\rangle=\phi\langle\psi\rangle_{f}, \quad \phi=\frac{V_{f}}{V}
$$

To derive the macroscopic governing equations, relations between the volumetric averages of derivatives and the derivatives of volumetric averages are needed. These relations are presented in a number of works $[22,26]$ and are 
known as the theorems of local volumetric average. For the case of a fixed solid, they can be written as:

$$
\begin{aligned}
& \langle\nabla \psi\rangle=\nabla\langle\psi\rangle+\frac{1}{V} \int_{A_{i}} \psi \mathbf{n} d S \\
& \langle\nabla \cdot \psi\rangle=\nabla \cdot\langle\psi\rangle+\frac{1}{V} \int_{A_{i}} \psi \cdot \mathbf{n} d S \\
& \left\langle\frac{\partial \psi}{\partial t}\right\rangle=\frac{\partial}{\partial t}\langle\psi\rangle
\end{aligned}
$$

where $A_{i}$ is the fluid-solid interfacial area and $\mathbf{n}$ is the unity normal vector oriented outward from the fluid into the solid phase (see figure 1). Note that these theorems are general and that, in particular, no length scale constraint is associated to their validity.

\subsection{Macroscopic continuity and momentum equations}

Applying the phase average operator to the microscopic equations (5) and (6), using the fact that the velocity vanishes at the fluid-solid interface and that the viscosity of the fluid is constant, the following result is obtained:

$$
\begin{aligned}
& \nabla \cdot\langle\mathbf{u}\rangle=0 \\
& -\nabla\langle p\rangle+\mu \nabla^{2}\langle\mathbf{u}\rangle=-\frac{1}{V} \int_{A_{i}}(\mu \nabla \mathbf{u}-p \mathbf{I}) \cdot \mathbf{n} d S
\end{aligned}
$$

In the analysis of fbws in porous media, the phase average velocity $\langle u\rangle$ is preferred to the intrinsic phase average velocity $\langle u\rangle_{f}$ because (i) it is representative of the mass fbw rate and (ii) it is solenoidal (Eq.(14)). However, the $i n$ trinsic phase average of the pressure $\langle p\rangle_{f}$ is preferably used, since it more closely corresponds to the measured value of the pressure. When the size of the REV $r_{0}$ is small compared to the characteristic length scale of variation of the macroscopic quantities $L$, but still sufficiently large such that the local average properties (porosity) are statistically meaningful, Carbonell and Whitaker [3] showed that:

$$
\frac{1}{V} \int_{A_{i}}\langle\psi\rangle_{f} \mathbf{n} d S=\langle\psi\rangle_{f} \frac{1}{V} \int_{A_{i}} \mathbf{n} d S
$$

Another important lemma is obtained, substituting $\psi=1$ in relation (11)

$$
\frac{1}{V} \int_{A_{i}} \mathbf{n} d S=-\nabla \phi
$$

Using Eqs. (10), (16), (17) and Gray's decomposition [8]:

$$
\psi=\langle\psi\rangle_{f}+\tilde{\psi}
$$

Eq. (15) can be rewritten in the form [27]:

$$
-\nabla\langle p\rangle_{f}+\frac{\mu}{\phi} \nabla^{2}\langle\mathbf{u}\rangle-\frac{\mu}{\phi} \nabla \phi \cdot \nabla\langle u\rangle_{f}=-\frac{1}{V_{f}} \int_{A_{i}}(\mu \nabla \tilde{\mathbf{u}}-\tilde{p} \mathbf{I}) \cdot \mathbf{n} d S
$$

The first viscous term that appears in Eq. (19) is the Brinkman correction. One can note that the viscosity associated with this term is the fluid viscosity $\mu$ divided by the porosity $\phi$. Thus, the effective viscosity is $\mu$ eff $=\mu / \phi$. The second viscous term is referred to as the second Brinkman correction by Ochoa-Tapia and Whitaker [13]. The closure of Eq. (19) is realized through the modeling of the term appearing in its right hand side which involves microscopic quantities.

\section{Homogeneous porous region:}

It has been shown $[27,17,18]$ that Eq. (19) reduces to a simple form for a viscous fbw in a homogeneous porous medium:

$$
\frac{\mu}{\phi} \nabla^{2}\langle\mathbf{u}\rangle-\mu \mathbf{K}^{-1}\langle\mathbf{u}\rangle=\nabla\langle p\rangle_{f}
$$

where $\mathbf{K}$ is the Darcy's law permeability tensor. Note that, since the porosity gradient is zero in the homogeneous porous region, the second Brinkman correction is null in this region. 


\section{Homogeneous fluid region:}

In the homogeneous fluid region, the right hand side of Eq. (19) is of course zero since there is no solid, and the second Brinkman correction is null since the porosity is constant (equal to 1). Furthermore, for a viscous fbw, Ochoa-Tapia and Whitaker [13] have shown that the point velocity is equal to the volume average velocity:

$$
\left.\langle\mathbf{u}\rangle\right|_{x}=\left.\mathbf{u}\right|_{x}
$$

when the size of the REV $r_{0}$ is small compared to the characteristic length scale of variation of the velocity. Thus, Eq. (19) reduces to the Stokes equation. Noting that the permeability is infinite in the homogeneous fluid region and that $\phi=1$, Eq. (20) is also valid in this region.

\section{Heterogeneous transition zone:}

Representing the boundary between the porous medium and the free fluid region as a sharp interface is only an idealization of a region where the properties of the medium encounter strong but nevertheless continuous variations. At the boundary between a porous medium and either a homogeneous fluid or a homogeneous solid, the porosity varies rapidly. Thus, the second Brinkman correction can be important. However, in this transition zone, the length scale constraint $r_{0}<<L$ used to obtain the second Brinkman correction (see Eq. (16)) is not necessarily valid, since the macroscopic quantities encounter strong variations on a short distance. As pointed out by Whitaker [27], there is no simple solution to the closure problem in this thin region of rapid variations.

Then, two different modeling approaches can be proposed: (i) to derive and solve a closure problem for $\tilde{u}$ and $\tilde{p}$ in the heterogeneous transition region. This approach has been used to derive jump conditions at the boundary between a porous catalyst and a homogeneous fluid $[28,15]$. However, to the authors knowledge, it has not been yet extended to the study of momentum transfer due to the complexity of the approach; (ii) to postulate the form of the closed equation, and to study the consequence of this choice. The second approach is followed in this study.

In order to propose a form to close Eq. (19) in the heterogeneous region, we suppose that no other forces than the ones acting in the homogeneous porous medium have to be taken into account in the heterogeneous transition zone. We assume that this heterogeneous transition zone of thickness $\delta$ is well represented by considering that the macroscopic properties of the medium $\phi$ and $\mathbf{K}$ are continuously varying from their values in the homogeneous porous region, to their values in the homogeneous flid region, and that the effects of the second Brinkman correction are taken into account through these variations of $\phi$ and $\mathbf{K}$. We have made this choice for different reasons. First, not taking into account the second Brinkman correction slightly simplify the problem. Furthermore, when studying variable porosity models for the heterogeneous transition zone, Goyeau et al. [7] were able to recover the jump conditions proposed by Ochoa-Tapia and Whitaker without the second Brinkman correction, whereas Ochoa-Tapia and Whitaker [14] could not while using the second Brinkman correction. With this assumption, equation (20) is valid everywhere. We are aware that this is a modeling choice. However, having chosen this particular model, we propose a rigorous method to determine the relevant form for the boundary conditions that must be applied at the interface separating the porous and free fluid media. Even though this choice is questionable, we show that the derived boundary conditions are consistent with observed data and they allow to improve the results of precedent models. Furthermore, the day the closure problem would be solved in the highly heterogeneous region, this method, applied here in a simple case, could be used.

To solve the system (14), (20), one needs to specify the exact variations of $\phi$ and $\mathbf{K}$ within the transition zone as well as the thickness of the transition zone $\delta$ in order to close the system. The objective of this study is to obtain a sufficiently accurate approximate solution of this system using the method of matched asymptotic expansions, without specifying a priori the functional dependence of $\phi$ and $\mathbf{K}$ within the transition zone, in order to construct acceptable jump conditions between the two homogeneous regions.

\section{One dimensional problem}

For the steady-state one-dimensional fbw process illustrated in figure 1, we assume that the variables are not $x$-dependent except for the pressure. From the continuity equation and the fact that the velocity vanishes at the upper wall (at $y=h$ ), it comes that the $y$-component of the velocity is zero. Thus, the set of equations (14), (20) reduces to:

$$
\begin{aligned}
& \frac{\mu}{\phi} \frac{d^{2}\langle u\rangle}{d y^{2}}-\mu K^{-1}\langle u\rangle=\frac{\partial\langle p\rangle_{f}}{\partial x} \\
& \langle u\rangle=0, \quad y=h \quad \text { no slip condition } \\
& \langle u\rangle \quad \text { is bounded as } \quad y \rightarrow-\infty
\end{aligned}
$$


where $u$ represents the $x$-component of the velocity vector and $K$ the permeability of the porous medium in the $x$-direction. Since the pressure gradient is zero in the $y$-direction (projection of the momentum equation on the $y$ axis), it follows that the pressure gradient $d\langle p\rangle_{f} / d x$ is a constant. The porosity $\phi$ and the permeability $K$ are only $y$ dependent.

\subsection{Non-dimensional form of the problem}

To make the system of equations (22)-(24) non-dimensional, the following scales are considered:

- $h$, height of the fluid channel: macroscopic length associated to the fluid region.

- $\sqrt{K_{p}}, K_{p}$ being the permeability of the homogeneous porous medium: macroscopic length associated to the homogeneous porous medium.

- $U_{\infty}$, asymptotic value of the volumetric average velocity in the porous medium as $y \rightarrow-\infty$.

- $\delta$, thickness of the boundary region.

Let us denote the non-dimensional variables by a superscript ${ }^{+}$. The following dimensionless variables are introduced:

$$
y^{+}=\frac{y}{h}, \quad U^{+}=\frac{\langle u\rangle}{U_{\infty}}, \quad K^{+}=\frac{K}{K_{p}}, \quad P^{+}=\langle p\rangle_{f} \frac{h}{U_{\infty} \mu}, \quad \phi^{+}=\phi
$$

The Darcy number $D a=K_{p} / h^{2}$ is also introduced. The dimensionless form of the boundary value problem (22)(24) is given by:

$$
\begin{aligned}
& \frac{1}{\phi^{+}} \frac{d^{2} U^{+}}{d y^{+2}}-\frac{1}{D a} \frac{U^{+}}{K^{+}}=\frac{d P^{+}}{d x^{+}} \\
& U^{+}=0, \quad y^{+}=1 \quad \text { no slip condition } \\
& U^{+} \rightarrow 1 \quad \text { when } \quad y^{+} \rightarrow-\infty
\end{aligned}
$$

\subsection{Method of matched asymptotic expansions}

The method of matched asymptotic expansions is applicable to the resolution of differential equations in which a small parameter is present. This method is classical $[30,29,4]$ and commonly used to study diffuse interface problems [5]. The main steps of the method, and in particular the origin of the matching conditions, are given only for completeness concern. In the present study, the small parameter is the ratio $\delta / h$ and will be noted $\epsilon$. This method consists in dividing the resolution domain in different sub-regions: an outer region, where the variables of the system are slowly varying, and an inner region, where these variables are rapidly varying. In our study, the heterogeneous transition zone is the inner region where the variables such as the porosity, the permeability and the velocity are rapidly varying, while each homogeneous region (porous medium and free fluid) is an outer region. Then a change of variable is made in the inner region, since variations of order one are expected to take place in this thin region and a new space variable is introduced:

$$
\bar{y}^{+}=\frac{y^{+}}{\epsilon}
$$

Let $f$ be any physical variable. Then, $\bar{f}$ is introduced in the inner region:

$$
\bar{f}\left(\bar{y}^{+}\right)=f\left(\frac{y^{+}}{\epsilon}\right)
$$

$\bar{f}\left(\bar{y}^{+}\right)$varies more slowly than $f\left(y^{+}\right)$in the inner region. Hereafter, the notation $f$ will designate any variable in the outer regions, while $\bar{f}$ will designate this variable in the inner region.

The differential equations are solved separately in each region, using an asymptotic expansion in $\epsilon$. For example, at first order, the solutions are sought for in the form:

$$
\begin{aligned}
& f\left(y^{+}, \epsilon\right)=f^{(0)}\left(y^{+}\right)+\epsilon f^{(1)}\left(y^{+}\right)+\mathcal{O}\left(\epsilon^{2}\right) \\
& \bar{f}\left(\bar{y}^{+}, \epsilon\right)=\bar{f}^{(0)}\left(\bar{y}^{+}\right)+\epsilon \bar{f}^{(1)}\left(\bar{y}^{+}\right)+\mathcal{O}\left(\epsilon^{2}\right)
\end{aligned}
$$


However, in order to solve completely the differential equations in each region, boundary conditions are needed. The missing boundary conditions are obtained by matching the solutions of the different regions using the following matching principles [30]:

$$
\begin{aligned}
\lim _{y^{+\rightarrow 0^{+}}} f\left(y^{+}, \epsilon\right) & =\lim _{\bar{y}^{+} \rightarrow+\infty} \bar{f}\left(\bar{y}^{+}, \epsilon\right) \hat{=} f_{i}^{+} \\
\lim _{y^{+} \rightarrow 0^{-}} f\left(y^{+}, \epsilon\right) & =\lim _{\bar{y}^{+} \rightarrow-\infty} \bar{f}\left(\bar{y}^{+}, \epsilon\right) \hat{=} f_{i}^{-}
\end{aligned}
$$

These relations mean that, for the study of the outer regions, the interface is viewed as a surface of discontinuity located in $0^{+}$(respectively $0^{-}$), and for the study of the inner region, the outer regions are supposed to be reached asymptotically in $+\infty$ and $-\infty . f_{i}^{+}$(respectively $f_{i}^{-}$) is the value of $f$ at the interface "side + " (respectively "side -", and is the same in the inner and the outer studies. Relations (33) and (34) are then made explicit. Using an asymptotic expansion of $f^{(0)}\left(y^{+}\right)$as $y^{+} \rightarrow 0^{+}$(respectively $y^{+} \rightarrow 0^{-}$), equation (31) can be rewritten at first order:

$$
f\left(y^{+}, \epsilon\right)=\lim _{y^{+\rightarrow 0^{ \pm}}} f^{(0)}\left(y^{+}\right)+\epsilon\left(\bar{y}^{+} \lim _{y^{+\rightarrow 0^{ \pm}}} \frac{d f^{(0)}}{d y^{+}}+\lim _{y^{+} \rightarrow 0^{ \pm}} f^{(1)}\left(y^{+}\right)+\right)+\mathcal{O}\left(\epsilon^{2}\right)
$$

Using equations (32), (33), (34) and (35), the following matching conditions are obtained at first order:

$$
\begin{aligned}
& \lim _{\bar{y}^{+} \rightarrow \pm \infty} \bar{f}^{(0)}=\lim _{y^{+} \rightarrow 0^{ \pm}} f^{(0)} \\
& \lim _{\bar{y}^{+} \rightarrow \pm \infty} \bar{f}^{(1)}=\lim _{y^{+} \rightarrow 0^{ \pm}} f^{(1)}+\bar{y}^{+} \lim _{y^{+} \rightarrow 0^{ \pm}} \frac{d f^{(0)}}{d y^{+}}
\end{aligned}
$$

Following this approach, matching conditions can be derived at higher order and for the derivatives of $f$ (e.g. Zwillinger [30]), Fouillet [6]). In this study, the following matching conditions will also be used:

$$
\begin{aligned}
& \lim _{\bar{y}^{+} \rightarrow \pm \infty} \frac{d \bar{f}^{(0)}}{d \bar{y}^{+}}=0 \\
& \lim _{\bar{y}^{+} \rightarrow \pm \infty} \frac{d \bar{f}^{(1)}}{d \bar{y}^{+}}=\lim _{y^{+} \rightarrow 0^{ \pm}} \frac{d f^{(0)}}{d y^{+}} \\
& \lim _{\bar{y}^{+} \rightarrow \pm \infty} \frac{d \bar{f}^{(2)}}{d \bar{y}^{+}}=\lim _{y^{+} \rightarrow 0^{ \pm}} \frac{d f^{(1)}}{d y^{+}}+\bar{y}^{+} \lim _{y^{+} \rightarrow 0^{ \pm}} \frac{d^{2} f^{(0)}}{d y^{+2}}
\end{aligned}
$$

Using these relations to match the solutions in the different regions, an approximate solution of the equations is obtained in the whole domain. Further explanations about this method can be found in $[30,29,4,6]$.

\subsubsection{Outer region}

To make an asymptotic expansion in $\epsilon$ of Eq. (26), we need to specify the variations of the porosity and the permeability in the outer region. At this point, we just assume that they are constant at least to second order:

$$
\begin{gathered}
\phi^{+}= \begin{cases}\phi_{p}+o\left(\epsilon^{2}\right), & y^{+}<0 \\
1+o\left(\epsilon^{2}\right), & y^{+}>0\end{cases} \\
\frac{1}{K^{+}}= \begin{cases}1+o\left(\epsilon^{2}\right), & y^{+}<0 \\
0+o\left(\epsilon^{2}\right), & y^{+}>0\end{cases}
\end{gathered}
$$

Such variations can be obtained by considering, for example, that the porosity and the inverse of the permeability vary as hyperbolic tangent functions:

$$
\begin{gathered}
\phi^{+}=\frac{1-\phi_{p}}{2} \tanh \left(\frac{y^{+}}{\epsilon}\right)+\frac{1+\phi_{p}}{2} \\
\frac{1}{K^{+}}=-\frac{1}{2} \tanh \left(\frac{y^{+}}{\epsilon}\right)+\frac{1}{2}
\end{gathered}
$$

For $y^{+}<0$, Eq. (26) reduces to:

$$
\frac{1}{\phi_{p}} \frac{d^{2}\left(U^{+(0)}+\epsilon U^{+(1)}+o(\epsilon)\right)}{d y^{+2}}-\frac{\left(U^{+(0)}+\epsilon U^{+(1)}+o(\epsilon)\right)}{D a}=\frac{d P^{+}}{d x^{+}}
$$


The analytical solutions of these two problems (at order 0 and order 1 ) are easily determined:

$$
\begin{aligned}
& U^{+(0)}\left(y^{+}\right)=1+C_{1}^{(0)} \exp \left(\sqrt{\frac{\phi_{p}}{D a}} y^{+}\right), \quad y^{+}<0 \\
& U^{+(1)}\left(y^{+}\right)=C_{1}^{(1)} \exp \left(\sqrt{\frac{\phi_{p}}{D a}} y^{+}\right), \quad y^{+}<0
\end{aligned}
$$

and the following relation is also obtained:

$$
-D a \frac{d P^{+}}{d x^{+}}=1
$$

For $0<y^{+}<1$, Eq. (26) reduces to:

$$
\frac{d^{2}\left(U^{+(0)}+\epsilon U^{+(1)}+o(\epsilon)\right)}{d y^{+2}}=\frac{d P^{+}}{d x^{+}}
$$

The analytical solutions of these two problems are given by:

$$
\begin{aligned}
& U^{+(0)}\left(y^{+}\right)=-\frac{1}{2 D a}\left(\left(y^{+}\right)^{2}-1\right)+C_{2}^{(0)}\left(y^{+}-1\right), \quad 0<y^{+}<1 \\
& U^{+(1)}\left(y^{+}\right)=C_{2}^{(1)}\left(y^{+}-1\right), \quad 0<y^{+}<1
\end{aligned}
$$

The four constants of integration $C_{1}^{(0)}, C_{1}^{(1)}, C_{2}^{(0)}$ and $C_{2}^{(1)}$ in the expressions for the velocity will be determined later, using the inner solutions and the matching conditions.

\subsubsection{Inner region}

In order to study the inner problem, the space variable (29) is used. Using this variable, Eq. (26) can be written as:

$$
\frac{1}{\bar{\phi}^{+}} \frac{1}{\epsilon^{2}} \frac{d^{2} \bar{U}^{+}}{d \bar{y}^{+2}}-\frac{1}{D a} \frac{\bar{U}^{+}}{\bar{K}}=\frac{d P^{+}}{d x^{+}}
$$

To solve this equation, we need to specify the functional dependence of the porosity and the permeability in the transition zone. At this point, we just assume that these functions are varying as $y / \delta$. It only implies that they are rapidly varying in the transition zone, as expected. Furthermore, since $\bar{y}^{+}=y^{+} / \epsilon=y / \delta$, it follows that $\bar{\phi}^{+}$and $\bar{K}^{+}$do not depend on $\epsilon$. Thus, the asymptotic expansion in $\epsilon$ of Eq. (52) reads:

$$
\frac{1}{\bar{\phi}^{+}} \frac{1}{\epsilon^{2}} \frac{d^{2}\left(\bar{U}^{+(0)}+\epsilon \bar{U}^{+(1)}+\epsilon^{2} \bar{U}^{+(2)}+o\left(\epsilon^{2}\right)\right)}{d \bar{y}^{+2}}-\frac{1}{D a} \frac{\left(\bar{U}^{+(0)}+o(1)\right)}{\bar{K}^{+}}=\frac{d P^{+}}{d x^{+}}
$$

and we have:

$$
\begin{array}{ll}
\text { Order 0: } & \frac{d^{2} \bar{U}^{+(0)}}{d \bar{y}^{+2}}=0 \\
\text { Order 1: } & \frac{d^{2} \bar{U}^{+(1)}}{d \bar{y}^{+2}}=0 \\
\text { Order 2: } & \frac{d^{2} \bar{U}^{+(2)}}{d \bar{y}^{+2}}=\bar{\phi}^{+} \frac{d P^{+}}{d x^{+}}+\frac{\bar{\phi}^{+}}{D a} \frac{\bar{U}^{+(0)}}{\bar{K}^{+}}
\end{array}
$$

The zeroth and first order analytical solutions can be expressed as:

$$
\begin{aligned}
& \bar{U}^{+(0)}=k_{1} \bar{y}^{+}+k_{2} \\
& \bar{U}^{+(1)}=k_{3} \bar{y}^{+}+k_{4}
\end{aligned}
$$

where the constants of integration $k_{i}, i \in\{1 . .4\}$ in the expressions for the velocity will be determined using the outer solutions and the matching conditions. 


\subsubsection{Zeroth order solution}

The inner solution of the problem is given by Eqs. (56), (57) and (58). In particular, these equations are valid for all $\bar{y}^{+} \in \mathbb{R}$. Accounting for the matching conditions (36), (38) and (39) applied to the velocity, it comes:

$$
\begin{aligned}
& k_{1}=0, \text { Thus } \bar{U}^{+(0)}=k_{2} \\
& k_{2}=\frac{1}{2 D a}-C_{2}^{(0)}=1+C_{1}^{(0)} \\
& k_{3}=\sqrt{\frac{\phi_{p}}{D a}} C_{1}^{(0)}=C_{2}^{(0)}=\left.\frac{d U^{+(0)}}{d y^{+}}\right|_{y^{+}=0}
\end{aligned}
$$

The system formed by Eqs. (60) and (61) is then solved:

$$
\begin{aligned}
C_{1}^{(0)} & =\frac{\sigma^{2}-2}{2\left(1+\sigma \sqrt{\phi_{p}}\right)} \\
C_{2}^{(0)} & =\sigma \sqrt{\phi_{p}} \frac{\sigma^{2}-2}{2\left(1+\sigma \sqrt{\phi_{p}}\right)}
\end{aligned}
$$

where $\sigma=1 / \sqrt{D a}$, following the nomenclature introduced by Beavers and Joseph [1].

Thus, we obtain the following boundary conditions at order 0 :

$$
\begin{aligned}
& \left.\langle u\rangle\right|_{y=0^{+}}=\left.\langle u\rangle\right|_{y=0^{-}} \\
& \left.\frac{d\langle u\rangle}{d y}\right|_{y=0^{+}}=\left.\frac{d\langle u\rangle}{d y}\right|_{y=0^{-}}
\end{aligned}
$$

These relations mean that, at order 0 , the volume average velocity $\langle u\rangle$ and the constraint built on the volume average velocities and the viscosity of the fluid $\mu d\langle u\rangle / d y$ are constant across the interface. The second boundary condition (Eq. (65)) is different from that used by Neale and Nader [11]. Indeed, Neale and Nader assume the continuity of the shear stress built using the effective viscosity ("effective" stress) whereas our development shows that it is the stress built using the viscosity of the fluid which is continuous at zeroth order across the interface. In fact this result is linked to the form of Eq. (20). If the ratio $\mu / \phi$ was inside the divergence operator, we would obtain a jump condition for the "effective" stress. It is not easy to conclude if one should or not put this ratio inside the divergence operator. Indeed, the closure expression given by Eq. (20) is obtained for a homogeneous porous medium, for which both formulations are equivalent since the porosity is constant. And for highly heterogeneous porous media, for which the difference is important, it is not possible to close the problem as discussed in Section 2.2. We made the choice to let the ratio $\mu / \phi$ outside the divergence operator because, using the up-scaling method, it appears first outside of the divergence. In the literature, both approaches can be found. Neale and Nader [11] keep this ratio $1 / \phi$ (or similarly $\mu_{\text {eff }}$ ) outside the divergence operator, while Goyeau et al. [7] put this ratio inside the divergence operator. In the study of Ochoa-Tapia and Whitaker $[13,14]$, the situation is even more complex since the ratio $\mu / \phi$ is first keep outside the divergence operator, before being introduce inside the divergence operator in order to arrange the Brinkman correction term into the form of a divergence to derive the jump conditions for the effective stress. We emphasize that the location of this ratio will remain a modeling choice until the closure problem will be solved in the highly heterogeneous region. However, once the choice is made to let this ratio outside of the divergence operator, our development shows that it is the stress built with the flid viscosity which is continuous across the interface at zeroth order and not the stress built with the effective viscosity.

The zeroth order solution of the problem is given in the outer regions by:

$$
\begin{aligned}
& \langle u\rangle=U_{\infty}\left(1+\frac{\sigma^{2}-2}{2\left(1+\sigma \sqrt{\phi_{p}}\right)} \exp \left(\sqrt{\frac{\phi_{p}}{K_{p}}} y\right)\right), \quad y \leq 0 \\
& \langle u\rangle=U_{\infty}\left(\frac{\sigma\left(\sigma+2 \sqrt{\phi_{p}}\right)}{2\left(1+\sigma \sqrt{\phi_{p}}\right)}+\sqrt{\phi_{p}} \frac{\sigma^{2}-2}{2\left(1+\sigma \sqrt{\phi_{p}}\right)}\left(\frac{y}{\sqrt{K_{p}}}\right)-\frac{1}{2}\left(\frac{y}{\sqrt{K_{p}}}\right)^{2}\right), \quad 0 \leq y \leq h
\end{aligned}
$$

This result is different from the one obtained by Neale and Nader [11], though very similar. The solution in the free fluid region $(y>0)$ is identical provided that their parameter $\beta=\sqrt{\mu_{\text {eff }} / \mu}$ is replaced everywhere by $\sqrt{\phi_{p}}$. 
However, this identification does not work for the solution in the porous medium region $(y<0)$ because their parameter $\beta$ should be replaced by $1 / \sqrt{\phi_{p}}$ in the exponential function. Furthermore, we note that only the second identification $\sqrt{\mu_{\text {eff }} / \mu}=1 / \sqrt{\phi_{p}}$ is consistent with our model (see Eq. (20)). This difference between the two results is a direct consequence of the boundary condition (65) which is different from their hypothesis to consider the continuity of the effective shear stress at the interface and is itself a consequence of our choice to keep the ratio $\mu / \phi$ outside the divergence operator.

\subsubsection{First order solution}

Accounting for the matching condition (37) applied to the velocity, the following result is obtained:

$$
k_{4}=C_{1}^{(1)}=-C_{2}^{(1)}
$$

This relation shows that, at order 1 in $\epsilon$, the volume average velocity across the interface is still constant. To obtain another relation between $C_{1}^{(1)}$ and $C_{2}^{(1)}$, we have to solve the inner problem at second order. Using Eq. (48), equation (56) can be written as follows:

$$
\frac{d}{d \bar{y}^{+}}\left(\frac{d \bar{U}^{+(2)}}{d \bar{y}^{+}}+\bar{y}^{+}\left(-\phi_{ \pm} \frac{d P^{+}}{d x^{+}}-\frac{\phi_{ \pm} U_{ \pm}}{D a K_{ \pm}}\right)\right)=\left(\bar{\phi}^{+}-\phi_{ \pm}\right) \frac{d P^{+}}{d x^{+}}+\left(\frac{\bar{\phi}^{+} \bar{U}^{+(0)}}{D a \bar{K}^{+}}-\frac{\phi_{ \pm} U_{ \pm}}{D a K_{ \pm}}\right)
$$

where $\psi_{ \pm}$are the constant asymptotic values of the corresponding quantities as $\bar{y}^{+} \rightarrow \pm \infty$. Accounting for the matching condition (40), it comes:

$$
\begin{aligned}
& \lim _{\bar{y}^{+\rightarrow+\infty}} \frac{d \bar{U}^{+(2)}}{d \bar{y}^{+}}=C_{2}^{(1)}-\frac{\bar{y}^{+}}{D a} \\
& \lim _{\bar{y}^{+} \rightarrow-\infty} \frac{d \bar{U}^{+(2)}}{d \bar{y}^{+}}=\sqrt{\frac{\phi_{p}}{D a}} C_{1}^{(1)}+\bar{y}^{+} \frac{\phi_{p}}{D a} C_{1}^{(0)}
\end{aligned}
$$

Equation (69) is first integrated from $y_{i}$, the exact position of the interface which is located somewhere in the heterogeneous transition zone, to $+\infty$. Using the asymptotic values at $+\infty$ and relation (70), one gets:

$$
C_{2}^{(1)}-\frac{d \bar{U}^{+(2)}}{d \bar{y}^{+}}\left(y_{i}\right)=\frac{-1}{D a} \int_{y_{i}}^{+\infty}\left(\bar{\phi}^{+}-\phi_{ \pm}\right)+\int_{y_{i}}^{+\infty}\left(\frac{\bar{\phi}^{+} \bar{U}^{+(0)}}{D a \bar{K}^{+}}\right)+\frac{y_{i}}{D a}
$$

Equation (69) is then integrated from $-\infty$ to $y_{i}$. Using the asymptotic values at $-\infty$ and relation (71), one gets:

$$
\frac{d \bar{U}^{+(2)}}{d \bar{y}^{+}}\left(y_{i}\right)-\sqrt{\frac{\phi_{p}}{D a}} C_{1}^{(1)}=\frac{-1}{D a} \int_{-\infty}^{y_{i}}\left(\bar{\phi}^{+}-\phi_{ \pm}\right)+\int_{-\infty}^{y_{i}}\left(\frac{\bar{\phi}^{+} \bar{U}^{+(0)}}{D a \bar{K}^{+}}-\frac{\phi_{p} \bar{U}^{+(0)}}{D a}\right)-y_{i} \frac{\phi_{p}}{D a}+y_{i} \frac{\phi_{p} \overline{U^{+(0)}}}{D a}
$$

By adding Eqs. (72) and (73), and using the fact that $\bar{U}^{+(0)}$ is constant in the transition zone, we obtain the following result:

$$
C_{2}^{(1)}-\sqrt{\frac{\phi_{p}}{D a}} C_{1}^{(1)}=\frac{\bar{U}^{+(0)}}{D a}\left(\frac{\bar{\phi}^{+}}{\bar{K}^{+}}\right)^{e x}-\frac{1}{D a}\left(\bar{\phi}^{+}\right)^{e x}
$$

where the excess quantity is defined by:

$$
\psi^{e x}=y_{i}\left(\psi_{-}-\psi_{+}\right)+\int_{-\infty}^{y_{i}}\left(\psi-\psi_{-}\right)+\int_{y_{i}}^{+\infty}\left(\psi-\psi_{+}\right)
$$

for any physical variable $\psi$. Equation (74) shows that, at order 1 in $\epsilon$, the constraint is not continuous.

In figure 2 we have plotted $\psi, \psi_{+}$and $\psi_{-}$. The corresponding excess quantity $\psi^{e x}$ is related to the shaded areas. Using this definition for the excess quantity, one can verify that its value is independent of the exact location of the interface $y_{i}$. This is important since the exact location of the interface cannot be known. Indeed, as already mentioned, the interface is only an ideal representation of a region with continuous but rapidly varying properties. For the model proposed by Beavers and Joseph, Saffman [20], Sahraoui and Kaviany [21] and Larson and Higdon [9] underlined the dependence of $\alpha$ on the exact location of the interface, whereas this precise location could not be 
accurately determined. Our development shows that such dependence does not exist, even though there does exist a dependence on the structure of the transition zone through the excess quantities as shown in the following section.

Then the system formed by Eqs. (68) and (74) is solved to determine $C_{1}^{(1)}$ and $C_{2}^{(1)}$ :

$$
C_{2}^{(1)}=\frac{\sigma^{2}}{\left(1+\sigma \sqrt{\phi_{p}}\right)}\left(\bar{U}^{+(0)}\left(\frac{\bar{\phi}^{+}}{\bar{K}^{+}}\right)^{e x}-\left(\bar{\phi}^{+}\right)^{e x}\right)=-C_{1}^{(1)}
$$

The first order solution of the problem is given in the outer regions by:

$$
\begin{array}{r}
\langle u\rangle=U_{\infty}\left(\left[\sqrt{\phi_{p}} \frac{\sigma^{2}-2}{2\left(1+\sigma \sqrt{\phi_{p}}\right)}+\frac{\delta}{h} \frac{\sigma}{1+\sigma \sqrt{\phi_{p}}}\left(\frac{\sigma\left(\sigma+2 \sqrt{\phi_{p}}\right)}{2\left(1+\sigma \sqrt{\phi_{p}}\right)}\left(\frac{\bar{\phi}^{+}}{\overline{K^{+}}}\right)^{e x}-\left(\bar{\phi}^{+}\right)^{e x}\right)\right]\left(\frac{y}{\sqrt{K_{p}}}-\sigma\right)\right. \\
\left.-\frac{1}{2}\left(\frac{y}{\sqrt{K_{p}}}\right)^{2}+\frac{\sigma^{2}}{2}\right), \quad 0 \leq y \leq h \quad(77) \\
\langle u\rangle=U_{\infty}\left(1+\left(\frac{\sigma^{2}-2}{2\left(1+\sigma \sqrt{\phi_{p}}\right)}+\frac{\delta}{h} \frac{\sigma^{2}}{\left(1+\sigma \sqrt{\phi_{p}}\right)}\left(-\frac{\sigma\left(\sigma+2 \sqrt{\phi_{p}}\right)}{2\left(1+\sigma \sqrt{\phi_{p}}\right)}\left(\frac{\bar{\phi}^{+}}{\bar{K}^{+}}\right)^{e x}+\left(\bar{\phi}^{+}\right)^{e x}\right)\right) \exp \left(\sqrt{\frac{\phi_{p}}{K_{p}} y}\right)\right), \\
y \leq 0 \quad(78)
\end{array}
$$

This solution verifies the following boundary conditions:

$$
\begin{aligned}
& \left.\langle u\rangle\right|_{y=0^{+}}=\left.\langle u\rangle\right|_{y=0^{-}} \\
& \left.\frac{\partial\langle u\rangle}{\partial y}\right|_{y=0^{+}}-\left.\frac{\partial\langle u\rangle}{\partial y}\right|_{y=0^{-}}=U_{\infty} \frac{\delta}{K_{p}}\left(\frac{\sigma\left(\sigma+2 \sqrt{\phi_{p}}\right)}{2\left(1+\sigma \sqrt{\phi_{p}}\right)}\left(\frac{\bar{\phi}^{+}}{\bar{K}^{+}}\right)^{e x}-\left(\bar{\phi}^{+}\right)^{e x}\right)
\end{aligned}
$$

We note that the volume average velocity is still continuous at first order in $\epsilon$, whereas the stress exhibits a discontinuity at first order in $\epsilon$. Since the velocity is continuous at the interface, this result can be expressed using the slip velocity defined here as $u_{B}=\left.\langle u\rangle\right|_{y=0}$ :

$$
\left.\langle u\rangle\right|_{y=0}=U_{\infty} \frac{\sigma\left(\sigma+2 \sqrt{\phi_{p}}\right)}{2\left(1+\sigma \sqrt{\phi_{p}}\right)}
$$

and the pressure gradient:

$$
-\frac{d\langle p\rangle_{f}}{d x}=\frac{U_{\infty} \mu}{K_{p}}
$$

Then, Eq. (80) reduces to:

$$
\left.\frac{\partial\langle u\rangle}{\partial y}\right|_{y=0^{+}}-\left.\frac{\partial\langle u\rangle}{\partial y}\right|_{y=0^{-}}=\left.\frac{\delta}{K_{p}}\left(\frac{\bar{\phi}^{+}}{\bar{K}^{+}}\right)^{e x}\langle u\rangle\right|_{y=0}+\frac{\delta}{\mu}\left(\bar{\phi}^{+}\right)^{e x} \frac{d\langle p\rangle_{f}}{d x}
$$

This expression is relevant to understand the phenomena to which the different excess quantities are related. However, in order to compare this result with the one proposed by Ochoa-Tapia and Whitaker [13], the jump is expressed using only the slip velocity $\left.\langle u\rangle\right|_{y=0}$. At first order, it comes:

$$
\left.\frac{\partial\langle u\rangle}{\partial y}\right|_{y=0^{+}}-\left.\frac{\partial\langle u\rangle}{\partial y}\right|_{y=0^{-}}=\left.\frac{\delta}{K_{p}}\left(\left(\frac{\bar{\phi}^{+}}{\bar{K}^{+}}\right)^{e x}-\left(\bar{\phi}^{+}\right)^{e x} \frac{2\left(1+\sigma \sqrt{\phi_{p}}\right)}{\sigma\left(\sigma+2 \sqrt{\phi_{p}}\right)}\right)\langle u\rangle\right|_{y=0}
$$

This result is similar to the one proposed by Ochoa-Tapia and Whitaker [13], and comparing with their result (4), we have:

$$
\beta_{p}=-\frac{\delta}{\sqrt{K_{p}}}\left(\left(\frac{\bar{\phi}^{+}}{\bar{K}^{+}}\right)^{e x}-\left(\bar{\phi}^{+}\right)^{e x} \frac{2\left(1+\sigma \sqrt{\phi_{p}}\right)}{\sigma\left(\sigma+2 \sqrt{\phi_{p}}\right)}\right)
$$

This result provides an explicit dependence of $\beta_{p}$ with the structure of the transition zone. One has to note that the jump condition (Eq. (83)) is built on the 'flid' stress, whereas the jump condition proposed by Ochoa-Tapia and 
Whitaker [13] was built on the 'effective' stress. It explains why the porosity does not appear on the left hand side of Eq. (83) unlike in the solution they proposed. It is also worth noting that the dependence of $\beta_{p}$ on the parameter $\delta / \sqrt{K_{p}}$ predicted by Ochoa-Tapia and Whitaker [13] naturally appears in our development. We also note the $\beta_{p}$ dependence on the other length scale ratio $\sigma$.

For the sake of simplicity, we introduce the following notations:

$$
\gamma=\left(\frac{\bar{\phi}^{+}}{\bar{K}^{+}}\right)^{e x}, \quad \lambda=\left(\bar{\phi}^{+}\right)^{e x}
$$

\subsubsection{Determination of the excess quantities}

The last unknowns of the problem are the values of the excess quantities $\gamma$ and $\lambda$ (see Eq. (86)). Because of its nature, an excess quantity can be either positive or negative. If the physical variables $\phi$ and $\phi / K$ were symmetric in the transition zone, the corresponding excess quantities would be null. Thus, the excess quantities are related to a loss of symmetry. They quantify the heterogeneity of the porous medium structure in the transition zone. In order to understand the phenomena to which they are associated, we go back to Eqs. (56) and (83). Eq. (56) gives the momentum balance inside the transition zone, whereas Eq. (83) characterizes the consequences of this momentum balance for the outer solution. $\lambda$ can be related to the porosity in front of the pressure gradient, which is constant, and $\gamma$ can be associated to the ratio $\phi / K$ in front of the velocity term. We recall that the velocity $\bar{U}^{+(0)}$ is constant, since the velocity is constant at zeroth order in the inner region. We deduce that an asymmetric porosity in the transition zone leads to an asymmetric effective viscosity $\mu_{\text {eff }}=\mu / \phi$ which influences the velocity gradient in the transition zone, the consequence being a velocity gradient jump for the outer solution. Thus, $\lambda$ allows to quantify the asymmetric effective viscosity of the transition region and we note that it is indeed the ratio $\lambda / \mu$ which appears in Eq. (83). Likewise, the asymmetry of the ratio $\phi / K$ results in an asymmetry in the drag, which also influences the velocity gradient in the transition zone, the consequence being a velocity gradient jump for the outer solution. Thus, $\gamma$ allows to quantify the asymmetric drag of the transition region. The difference between the two phenomena allows to understand why the $\sigma$ dependence of $\beta$ only appears in front of $\lambda$. $\gamma$ being associated to an asymmetry of the drag at constant velocity, it cannot keep the memory of the height of the channel $\left(\sigma=h / \sqrt{K_{p}}\right)$, whereas $\lambda$ can, since it is related to the pressure term. Yet, these two phenomena are intimately linked, and we cannot think of an experiment that would allow us to determine these two quantities independently.

To obtain an idea of the order of magnitude of the excess quantities $\gamma$ and $\lambda$, we compute their values for different variations of $\phi$ and $K$ in the transition zone. First, we assume that the variations of the porosity and of the inverse of the permeability are linear. We obtain:

$$
\lambda=0, \quad \gamma=\frac{1-\phi_{p}}{6}
$$

If we assume a variation of these quantities following a hyperbolic tangent, we obtain:

$$
\lambda=0, \quad \gamma=\frac{1-\phi_{p}}{2}
$$

To obtain a non zero value for $\lambda$, one has to consider a loss of symmetry for the porosity. This is not the case for $\gamma$, since its value can be non zero for symmetric variations of $\phi$ and $1 / K$.

If we assume that the porosity is bounded in the heterogeneous region by its two asymptotic values in the homogeneous region (no material storage in the transition zone), it follows that the corresponding excess quantity is bounded by:

$$
-\frac{1-\phi_{p}}{2} \leq \lambda \leq \frac{1-\phi_{p}}{2}
$$

If we make the same assumption for the inverse of the permeability, it follows that:

$$
-\frac{\phi_{p}}{2} \leq \gamma \leq 1-\frac{\phi_{p}}{2}
$$

We cannot provide better predictions for the values of the excess quantities, since we do not know the functional dependence of the porosity and permeability within the transition zone. However, the above relations show that they should be of the order of one, either negative or positive, if we assume no material storage in the transition zone. 


\section{Comparisons with experimental data}

Beavers and Joseph [1] carried out comparisons between theory and experiment in terms of the fractional increase in mass fbw rate $\Phi$ due to the presence of the porous wall:

$$
\Phi=\frac{Q_{p}-Q_{i}}{Q_{i}}
$$

where $Q_{p}$ is the mass fbw rate within the free fluid region for a given porous medium, while $Q_{i}$ is the mass fbw rate for an impermeable wall. The comparison with the experimental results is performed using the characteristics of the porous materials used by Beavers and Joseph [1] in their experiments as reported by Goyeau et al. [7] and recalled in Table 1. According to theoretical studies [9, 10,21], $\delta$ is of the order of the average pore diameter of the porous medium $d_{p}$. We use this assumption to compute the parameter $\delta / \sqrt{K_{p}}$. The obtained values are presented on Table 1. Using only the zeroth order solution for the velocity, the following result is obtained:

$$
\Phi^{(0)}=\frac{3\left(\sigma+2 \sqrt{\phi_{p}}\right)}{\sigma\left(1+\sigma \sqrt{\phi_{p}}\right)}
$$

This expression is identical to the one derived by Beavers and Joseph [1] after identifying their parameter $\alpha$ with $\sqrt{\phi_{p}}$. However, this zeroth order result is not sufficient. Indeed, whereas the four foametal materials have the same porosity (see Table 1), Beavers and Joseph use four different values of $\alpha$ to fit their experimental results. Using the first order solution for the velocity, one obtains:

$$
\Phi^{(1)}=\Phi^{(0)}+\frac{\delta}{\sqrt{K_{p}}} \frac{1}{\sigma \sqrt{\phi_{p}}+1}\left(\frac{6}{\sigma} \lambda-\sigma \Phi^{(0)} \gamma\right)
$$

In figures 3-6, we compare the analytical solutions given by equations (92)-(93) with the experimental data of Beavers and Joseph [1].

For the first foametal material (figure 3), good agreement between theory and experiment is obtained at zeroth order $\Phi^{(0)}$. For the three other foametals materials (figure 4), the zeroth order solution is not sufficiently accurate, even though the order of magnitude is correct. Better agreement is obtained when considering the first order solution $\Phi^{(1)}$, for values of $\gamma$ ranging from -0.05 to 0.16 , and considering $\lambda=0$. This result is consistent with the fact that $\gamma$ should be in the range $[-0.39,0.61]$ (Eq. (90)) and that its sign may be either positive or negative due to the nature of an excess quantity, as discussed in the previous section. We indicate that we always first tried to fit the experimental data with $\lambda=0$, since a non zero value of $\lambda$ means a loss of symmetry, which is not the case for $\gamma$ as pointed out in the previous section.

For the Aloxite experiments the zeroth order solution does not fit at all the experiments (figures 5- 6). However, good agreement is obtained when considering the first order solution $\Phi^{(1)}$, for values of $\gamma$ ranging from -0.18 to -0.3 , and considering $\lambda=0$. These values of $\gamma$ are of the same order as the one used to fit the foametal experiments. The significant difference comes from the value of the ratio $\delta / \sqrt{K_{p}}$ which is greater for the aloxite blocks. These first order results, obtained with $\lambda=0$, are very similar to the ones proposed by Ochoa-Tapia and Whitaker [14], as can be seen in figures $5-6$.

One can note that for the aloxite experiment, the slope of the fractional increase in mass fbw rate $\Phi$ versus $\sigma$ is still not accurately predicted by these two models. A better result is obtained by taking non zero values for $\lambda$, which means that we consider that the porosity is no longer symmetric in the transition zone. However, the values of $\lambda$ that best fit the experimental values ( 2 and 5 ) are out of the expected range predicted by Eq. (89). This could be explained if we suppose that the thickness of the heterogeneous transition zone has been underestimated by a factor 8 for the aloxite 1 and by a factor 20 for the aloxite 2 . This would imply significant heterogeneous regions at the interface of the porous material. It could be the consequence of the structure of the aloxite material which has probably a very irregular pore distribution close to the interface [1]. The other possible explanation is related to the validity of the assumption that no material storage occurs in the transition zone (Eq. (89)). It might be possible that, during the machining, the material suffered at its surface, leading to a decrease of the material porosity beneath the interface. This would imply large values of $\lambda$. The two combined effects (significant size of the heterogeneous region and material storage) could explain the very large value of $\lambda$ obtained for the aloxite 2 . To conclude on this point, more informations about the surface micro structure of the materials used for the experiments are necessary.

In order to make a quantitative comparison with the values of $\beta$ estimated by Ochoa-Tapia and Whitaker [14], the values for $\beta_{p}$ (Eq. 85), obtained once $\lambda$ and $\gamma$ have been adjusted to fit the Beavers and Joseph experiment [1], 
are shown in table 2. When $\lambda$ is non zero (Aloxite experiments), $\beta_{p}$ is no longer a constant and depends on $\sigma$. Thus, the extremum values of $\beta_{p}$ obtained for $\sigma=20$ and $\sigma=100$ are presented. As can be seen on this table, the values obtained for $\beta_{p}$ are different from the one proposed by Ochoa-Tapia and Whitaker [14] to fit the Beavers and Joseph experiment [1]. It is not surprising, since, as mentioned before, the stress jump conditions are not built on the same stress.

These results show that the increase in mass fbw rate $\Phi$ is very sensitive to the value of the excess quantities, which is unfortunately unknown and related to the surface of the porous medium. We recall that Beavers et al. [2] found that the value of the slip coefficient $\alpha$ for a certain material doubled after its surface was remachined. Sahraoui and Kaviany [21] also established that the surface structure (structural non-uniformities at the surface) influences significantly the slip coefficient $\alpha$. They concluded that $\alpha$ was mainly a surface property. This strong dependence is highlighted by our developments. Furthermore, our analysis directly relates these excess quantities to the variations of the porosity and of the permeability in the transition zone. Therefore, if, by any mean (experimentally or theoretically), we could have access to this information, we could determine their values.

Finally, we emphasize that thanks to this analysis, unlike the studies of Ochoa-Tapia and Whitaker [14] and Goyeau et al. [7], it is no longer required to solve Eq. (22) in the whole channel to obtain the increase in mass fbw rate for given variations of porosity and permeability in the heterogeneous transition zone, and to start again the simulation for different values of $h$. One just needs to compute the excess quantities corresponding to the chosen variations of porosity and permeability.

\section{Discussion}

The first subject of discussion involves the ratio $\mu / \phi=\mu_{\text {eff. }}$ In most studies, this ratio is kept outside of the divergence operator of the Darcy-Brinkman equation (see Eq. (20)), whereas the continuity of the 'effective' stress is considered. Our analysis shows that this is inconsistent, even though it is not easy to conclude where one should put this ratio. Indeed, as already pointed out, the closure of the averaging problem is always obtained for homogeneous regions for which both formulations are equivalent since the porosity is constant. Furthermore, these two formulations leads to very similar results in terms of the increase in mass fbw rate. And in both cases, one needs to adjust an a priori unknown parameter to fit the experimental data. Thus, it is not possible with these experiments to conclude. The study of highly heterogeneous porous medium might give an answer to this question.

Another discussion concerns the second Brinkman correction. Since this term involves the gradient of the porosity, it is still not easy to conclude if one needs to introduce this term, or if this term is already taken into account by considering variable porosity and permeability in the heterogeneous transition zone. However, we can say that this term is not essential to recover the jump conditions. And it might even be better not to introduce it, since Goyeau $e t$ al. [7] were able to recover the jump conditions without the second Brinkman correction, whereas Ochoa-Tapia and Whitaker [14] could not.

\section{Conclusion}

In this paper, we have studied the single phase Poiseuille fbw over a permeable block and we have derived the boundary conditions that must be applied at the interface between the porous medium and the free fluid. Based on the observation that a sharp interface is only an idealization of a region where the properties of the medium encounter strong but nevertheless continuous variations, a heterogeneous transition zone is introduced between the two homogeneous regions (i.e. porous medium and free flid). It is shown that the same volume averaged transport equation can be used to describe the two homogeneous regions, and it is assumed that this equation still holds in the heterogeneous transition zone by considering variable porosity and permeability. Then, the problem is solved using the method of matched asymptotic expansions, without specifying a priori the functional dependence of the porosity and permeability in the transition zone. The resolution of this problem proves that, if the validity of the single volume averaged transport equation is assumed in the whole domain, then the velocity is continuous across the interface (79), whereas the stress is discontinuous (83). It also proves that, it is a stress jump condition built using the fluid viscosity that has to be considered at the interface, whereas previous studies (e.g. $[11,13])$ suggested to use a stress jump condition built using the effective viscosity of the porous medium. Furthermore, in previous studies, the stress jump condition was only related to the slip velocity through a stress jump parameter [13]. The analytical development shows that the stress jump condition is also related to the pressure gradient, through a second jump parameter (see Eq. (83)). This study provides an explicit dependence of the stress jump coefficient on the 
variations of the porosity and permeability in the transition zone, expressed through two excess quantities which are actually surface properties. Thus, the stress jump coefficient is directly linked to the surface properties of the porous medium. An estimation of these excess quantities has been proposed. Good agreement is obtained between theory and the Beavers and Joseph [1] experiment by adjusting the values of these two excess quantities. In particular, we were able to correctly predict the slope of the fractional increase in mass fbw rate $\Phi$ versus the non-dimensional height of the free fluid region $\sigma$ for the aloxite experiments, a result that other models cannot recover. Even though we had to adjust the values of the excess quantities to obtain good agreement with the available data, our analysis directly relates these excess quantities to the variations of the porosity and permeability in the transition zone (see Eq. (75)). Thus, further work should focus on the means (either experimental or theoretical) of evaluating a priori the values of these excess quantities for the surface of a given material. If such informations were available, we would be able to predict the stress jump coefficient without resorting to experiments. Furthermore, this study suggests a methodology to derive boundary conditions between a free fluid and a porous medium and could be used to derive boundary conditions for other transport phenomena: heat, species,...

\section{References}

[1] G.S. Beavers and D.D. Joseph. Boundary conditions at a naturally permeable wall. J. Fluid Mech., 30:197-207, 1967.

[2] G.S. Beavers, E.M. Sparrow, and B.A. Masha. Boundary conditions at a porous surface which bounds a fluid fbw. AIChE J., 20:596-597, 1974.

[3] R. G. Carbonell and S. Whitaker. Fundamentals of transport phenomena in porous media. M. Nijhoff, Dordrecht, 1984.

[4] M. Van Dyke. Perturbation methods in fluid mechanics. The Parabolic Press, Standford, California, 1975.

[5] H. Emmerich. The Diffuse Interface Approach in Materials Science. Springer, 2003.

[6] C. Fouillet. Généralisation à des mélanges binaires de la méthode du second gradient et application à la simulation numérique directe de l'ébullition nucléée. PhD thesis, Univ. Paris 6, 2003.

[7] B. Goyeau, D. Lhuillier, D. Gobin, and M. G. Velarde. Momentum transport at a flidd-porous interface. Int. J. Heat Mass Transfer, 46:4071-4081, 2003.

[8] W. G. Gray. A derivation of the equations for multiphase transport. Chem. Engng. Sci., 30:229-233, 1975.

[9] R.E. Larson and J.J.L Higdon. Microscopic fbw near the surface of two-dimensional porous media. Part 1. Axial fbw. J. Fluid Mech., 166:449-472, 1986.

[10] R.E. Larson and J.J.L Higdon. Microscopic fbw near the surface of two-dimensional porous media. Part 2. Transverse fbw. J. Fluid Mech., 178:119-136, 1987.

[11] G. Neale and W. Nader. Practical significance of Brinkman's extension of Darcy's law: Coupled parallel fbws within a channel and a bounding porous medium. Can. J. Chem. Eng., 52:475-478, 1974.

[12] D. A. Nield. The limitations of the Brinkman-Forchheimer equations in modeling fbw in a saturated porous medium and at an interface. Int. J. Heat and Fluid Flow, 12(3):269-272, 1991.

[13] J. A. Ochoa-Tapia and S. Whitaker. Momentum transfer at the boundary between a porous medium and a homogeneous fluid - I. Theoretical development. Int. J. Heat Mass Transfer, 38(14):2635-2646, 1995.

[14] J. A. Ochoa-Tapia and S. Whitaker. Momentum transfer at the boundary between a porous medium and a homogeneous fluid - II. Comparison with experiment. Int. J. Heat Mass Transfer, 38(14):2647-2655, 1995.

[15] F.J. Valdes Parada and J.A. Ochoa Tapia. Jump condition at the boundary between a porous catalyst and a homogeneous fluid. In Proc. of the 4th Int. Conf. on Computational Heat and Mass Transfer, volume 1, pages 323-326, 2005.

[16] D. Poulikakos and M. Kazmierczak. Forced convection in a duct partially filled with a porous material. J. Heat Transfer, 109:653-662, 1987. 
[17] M. Quintard and S. Whitaker. Transport in order and disordered porous media - I. The cellular average and the use of weighting functions. Transp. Porous Media, 14:163-177, 1994.

[18] M. Quintard and S. Whitaker. Transport in order and disordered porous media - II. Generalized volume averaging. Transp. Porous Media, 14:179-206, 1994.

[19] N. Rudraiah. Coupled parallel fbws in a channel and a bounding porous medium of finite thickness. J. Fluids Eng., 107:322-329, 1985.

[20] P.G. Saffman. On the boundary condition at the surface of a porous medium. Stud. Appl. Math., L(2):93-101, 1971.

[21] M. Sahraoui and M. Kaviany. Slip and no-slip velocity boundary conditions at interface of porous, plain media. Int. J. Heat Mass Transfer, 35(4):927-943, 1992.

[22] J. C. Slattery. Momentum, Energy and Mass Transfer in Continua. McGraw-Hill, New York, 1972.

[23] K. Vafai and S.J. Kim. Fluid mechanics of the interface region between a porous medium and a fluid layer - an exact solution. Int. J. Heat and Fluid Flow, 11(3):254-256, 1990.

[24] K. Vafai and S.J. Kim. On the limitations of the Brinkman-Forchheimer-extended Darcy equation. Int. J. Heat and Fluid Flow, 16(1):11-15, 1995.

[25] K. Vafai and R. Thiyagaraja. Analysis of fbw and heat transfer at the interface region of a porous medium. Int. J. Heat Mass Transfer, 30:1391-1405, 1987.

[26] S. Whitaker. Advances in theory of fluid motion in porous media. Ind. Eng. Chem., 61:14-28, 1969.

[27] S. Whitaker. Flow in porous media. I: A theoretical derivation of Darcy's law. Transport in Porous Media, $1: 3-25,1986$.

[28] B. D. Wood, M. Quintard, and S. Whitaker. Jump conditions at non-uniform boundaries: the catalytic surface. Chem. Eng. Sc., 55:5231-5245, 2000.

[29] R. Kh. Zeytounian. Les modèles Asymptotiques de la mécanique des fluides I, Lecture Notes in Physics. Springer-Verlag, Berlin, 1986.

[30] D. Zwillinger. Handbook of differential equations. Academic Press, Boston, 1989.

\begin{tabular}{ccccc}
\hline Porous specimen & Permeability $K_{p}$ & Pore size & Porosity $\phi_{p}$ & $\delta / \sqrt{K_{p}}$ \\
\hline Foametal & $7.1 \times 10^{-9}$ & - & 0.78 & - \\
Foametal A & $9.7 \times 10^{-9}$ & $4.06 \times 10^{-4}$ & 0.78 & 4.12 \\
Foametal B & $3.94 \times 10^{-8}$ & $8.64 \times 10^{-4}$ & 0.78 & 4.35 \\
Foametal C & $8.2 \times 10^{-8}$ & $1.14 \times 10^{-3}$ & 0.79 & 3.98 \\
Aloxite 1 & $6.45 \times 10^{-10}$ & $3.30 \times 10^{-4}$ & 0.58 & 12.99 \\
Aloxite 2 & $1.6 \times 10^{-9}$ & $6.86 \times 10^{-4}$ & 0.52 & 17.15 \\
\hline
\end{tabular}

Table 1: Geometrical characteristics of the porous specimens used in the Beavers and Joseph experiments 


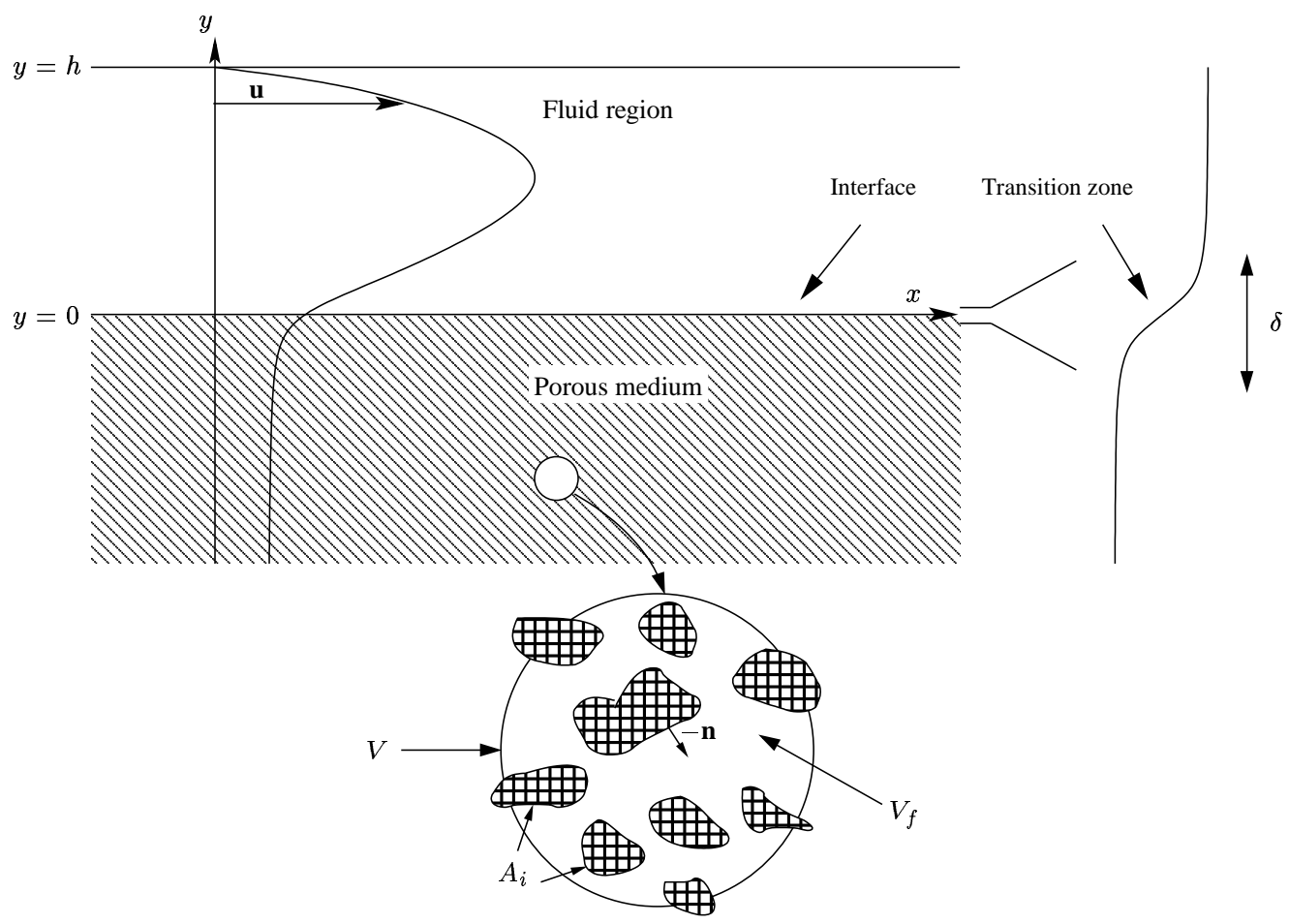

Figure 1: Fluid fbw parallel to an infinite porous medium

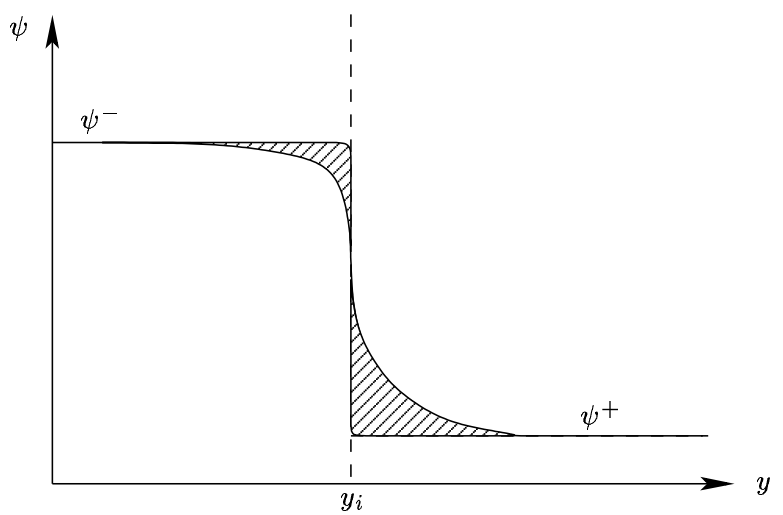

Figure 2: Determination of an excess function

\begin{tabular}{ccccc}
\hline Porous specimen & $\gamma$ & $\lambda$ & $\beta_{p}$ & $\beta$ (Ochoa-Tapia and Whitaker) \\
\hline Foametal & 0 & 0 & 0 & 0.6 \\
Foametal A & -0.05 & 0 & 0.206 & 0.7 \\
Foametal B & 0.06 & 0 & -0.261 & 0 \\
Foametal C & 0.16 & 0 & -0.637 & -1 \\
Aloxite 1 (first result) & -0.3 & 0 & 3.89 & 1.47 \\
Aloxite 1 (second result) & -0.25 & 2 & {$[5.21-3.64]$} & 1.47 \\
Aloxite 2 (first result) & -0.25 & 0 & 4.28 & 1.47 \\
Aloxite 2 (second result) & -0.05 & 5 & {$[7.02-2.10]$} & 1.47 \\
\hline
\end{tabular}

Table 2: Comparison of the stress jump parameters $\beta_{p}$ and $\beta$ 
Fractional excess flow rate $\Phi$ versus $\sigma$ for the first Foametal

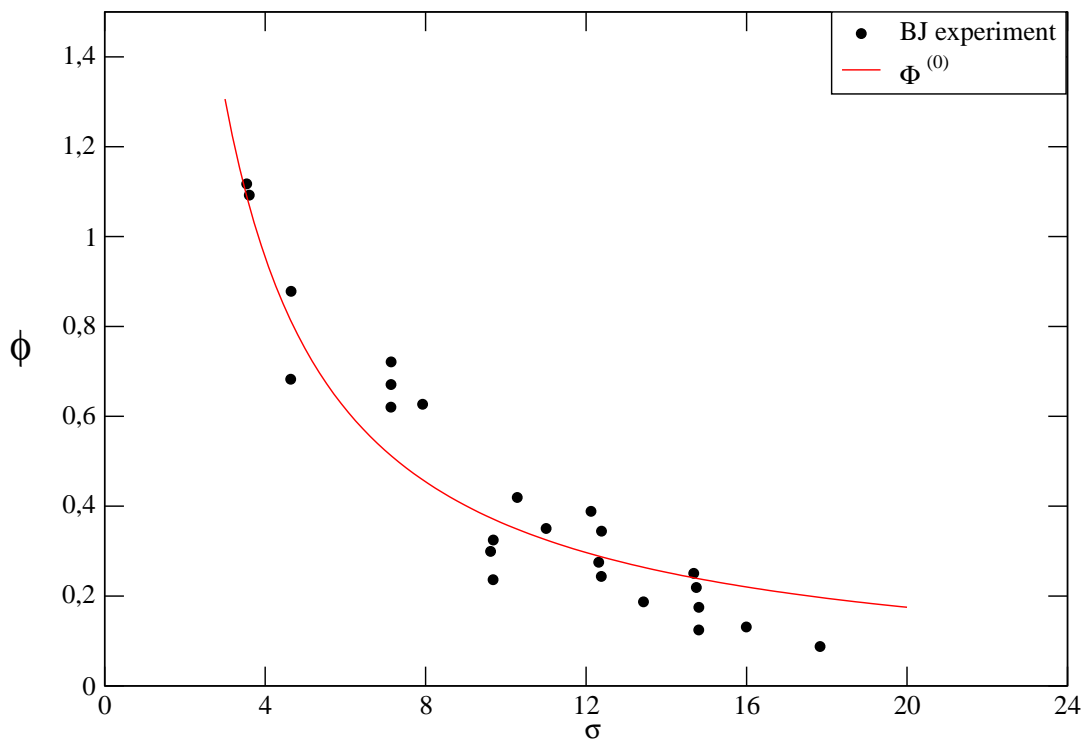

Figure 3: Comparison between theories and experiments for the first foametal.

\section{Fractional excess flow rate $\Phi$ versus $\sigma$ for Foametal}

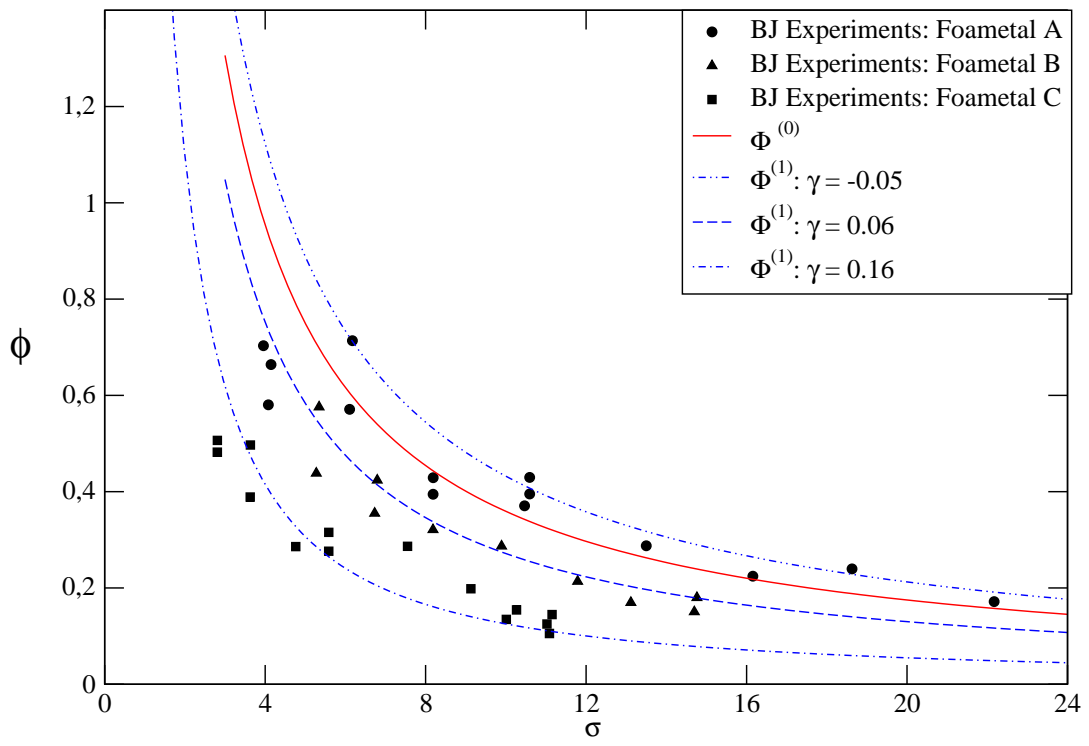

Figure 4: Comparison between theories and experiments for foametals A, B and C. 
Fractional excess flow rate $\Phi$ versus $\sigma$ for Aloxite 1

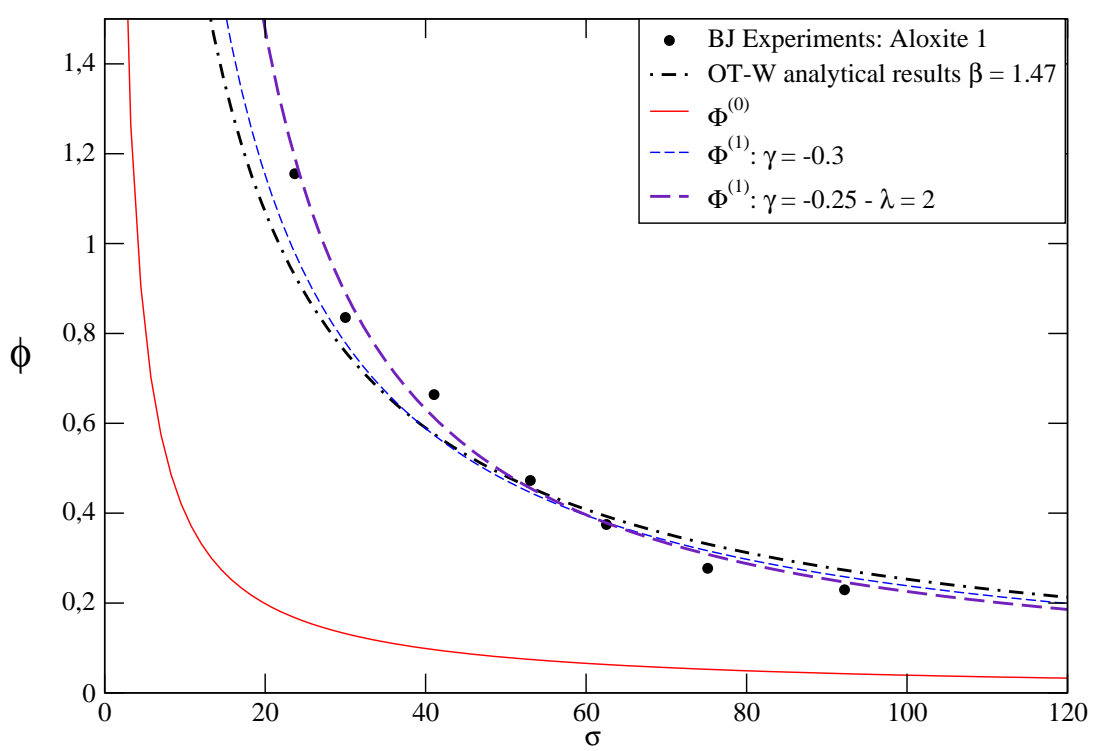

Figure 5: Comparison between theories and experiments for Aloxite 1.

Fractional excess flow rate $\Phi$ versus $\sigma$ for Aloxite 2

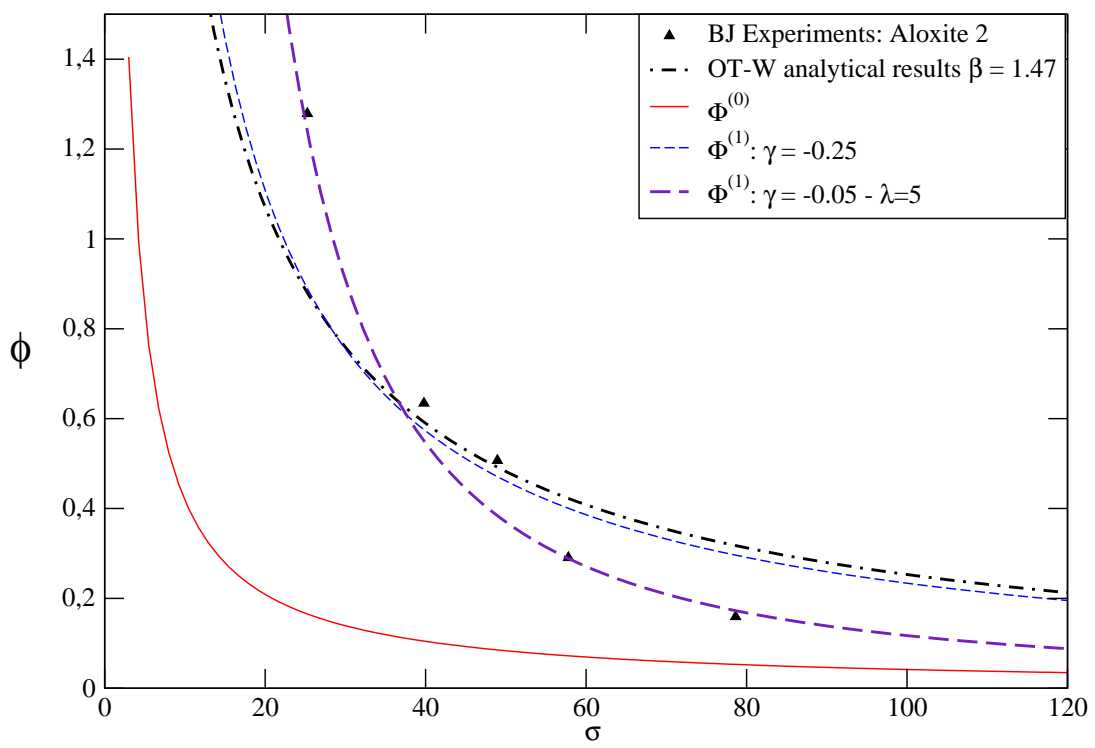

Figure 6: Comparison between theories and experiments for Aloxite 2. 